\title{
ARTICLE OPEN \\ An efficient descriptor model for designing materials for solar cells
}

\author{
Fahhad H Alharbi ${ }^{1,2}$, Sergey N Rashkeev ${ }^{2}$, Fedwa El-Mellouhi ${ }^{2}$, Hans P Lüthi ${ }^{3}$, Nouar Tabet ${ }^{1,2}$ and Sabre Kais ${ }^{1,2}$
}

An efficient descriptor model for fast screening of potential materials for solar cell applications is presented. It works for both excitonic and non-excitonic solar cells materials, and in addition to the energy gap it includes the absorption spectrum $(a(E))$ of the material. The charge transport properties of the explored materials are modelled using the characteristic diffusion length $\left(L_{\mathrm{d}}\right)$ determined for the respective family of compounds. The presented model surpasses the widely used Scharber model developed for bulk heterojunction solar cells. Using published experimental data, we show that the presented model is more accurate in predicting the achievable efficiencies. To model both excitonic and non-excitonic systems, two different sets of parameters are used to account for the different modes of operation. The analysis of the presented descriptor model clearly shows the benefit of including $a(E)$ and $L_{\mathrm{d}}$ in view of improved screening results.

npj Computational Materials (2015) 1, 15003; doi:10.1038/npjcompumats.2015.3; published online 25 November 2015

\section{INTRODUCTION}

There has been a remarkable thrust towards developing costeffective photovoltaics in the past two decades. ${ }^{1-5}$ Different materials and device concepts have been deployed and the highest achieved conversion efficiency so far is $44.7 \%$ by quadruple junction using III-V materials. ${ }^{6}$ As for the market, it is dominated by the conventional Si solar cells. Nonetheless, dye-sensitised solar cells, organic photovoltaics (OPV) and the recently emerged hybrid perovskite solar cells could become more cost effective and competitive if produced at large scale. ${ }^{7}$

In principle, an efficient single-junction solar cell can be made of any semiconducting material with an energy gap $\left(E_{\mathrm{g}}\right)$ ranging between 1.0 and $1.7 \mathrm{eV}$ and with reasonable transport to allow the photogenerated carrier to be collected. ${ }^{4,8-11}$ Hence, many organic and inorganic semiconductors have been used to make solar cells. ${ }^{4,11}$ The selection was mostly based on known materials as, till recently, experimental data were the main source for screening materials for solar cells. Despite the rich data, this certainly limits the screening space. However, the sophisticated computational capabilities have provided an alternative route to explore new materials for solar cells much beyond the rich experimental data. There are many initiatives in this regard. Among the most noticeable ones is the Clean Energy Project at Harvard University. ${ }^{12-14}$ It is a high-throughput discovery and design program for the next generation of OPV materials. By 2013, 2.3 million of organic molecules and polymers were analysed using $>150$ million density functional theory calculations to assess their applicability for solar cells. ${ }^{14}$

The Clean Energy Project, like other initiatives, ${ }^{15-17}$ is based on atomistic calculations, which are then fed into empirical descriptor models to assess the potential of the studied material for photovoltaics. The commonly used descriptor model, at least within the OPV community, is the one proposed by Scharber, ${ }^{18}$ a one-parameter model based on the computed $E_{g}$ in which the open-circuit voltage $\left(V_{\text {oc }}\right)$ is assumed to be a fixed reduction of $E_{\mathrm{g}}$ defined (by the Scharber model) as the difference between the highest occupied molecular orbital of the donor and the lowest unoccupied molecular orbital of the acceptor. The short-circuit current $\left(J_{\mathrm{sc}}\right)$ is estimated as a fraction of the current resulting from absorbing all incident photons above $E_{\mathrm{g}}$, whereas the fill factor (FF) is set to a fixed value. Usually, the FF and the scaling parameter for $J_{\text {sc }}$ are both set to a value of 0.65 . Although these approximations for $V_{\text {oc }}$ and FF appear reasonable, the assumption that all the photons above $E_{\mathrm{g}}$ are absorbed and a fraction of them is extracted as current is an extreme oversimplification. These assumptions ignore the inhomogeneity of the absorption spectrum. Furthermore, it assumes that the transport is highly efficient and that the diffusion length is much larger than the absorption length so that the detailed balance fraction of the photogenerated carriers can be collected. Practically, it is important to consider in more detail the absorption spectrum and the transport limitations.

In this paper, we propose a descriptor model where the absorption spectrum $(a(E))$ is obtained from the same electronic structure calculations used to determine $E_{\mathrm{g}} . E$ is the photon energy in $\mathrm{eV}$, which is used as the unit for energy throughout in this paper. These are the only atomistic calculations needed here. In addition, the transport is characterised by the diffusion length $\left(L_{\mathrm{d}}\right)$, which is a measure for the mean distance that an excited carrier can cross through random diffusion before recombining. Calculating $L_{d}$ needs lengthy calculations, which would make combinatorial screening prohibitively expensive. To avoid this, each material is given a value for $L_{d}$, which is characteristic for the family of compounds it belongs to.

The focus in this paper is on OPV as in the Scharber model. Nonetheless, the same model is applicable to other photovoltaics technologies. Yet, due to the slightly different modes of operation between excitonic (such as OPV) and non-excitonic (e.g., inorganic

\footnotetext{
${ }^{1}$ College of Science and Engineering, Hamad Bin Khalifa University, Doha, Qatar; ${ }^{2}$ Qatar Environment and Energy research Institute (QEERI), Hamad Bin Khalifa University, Doha, Qatar and ${ }^{3}$ Department of Chemistry and Applied Bioscience, ETH Zurich, Zurich, Switzerland.

Correspondence: FH Alharbi (falharbi@qf.org.qa)

Received 11 June 2015; revised 20 August 2015; accepted 28 August 2015
} 
semiconductor cells) solar cells, two distinct sets of parameters should be used. For non-excitonic solar cells, the binding energy of excitons is small and hence the exciton can be dissociated thermally or by potential gradient. On the other hand, in excitonic cells, where the binding energy is large, the heterojunction band offset is needed to dissociate excitons. Thus, a considerable additional loss in the voltage is unavoidable. Therefore, it is essential to make distinction between these two classes of cells.

We intend to apply our model for large-scale virtual screening of organic compounds, where the absorption spectra fluctuate considerably. Hence, we expect that by taking into account the details of their absorption spectrum, we will be able to better discriminate between candidate compounds. The initial validation analysis clearly shows the merit of including $a(E)$ and $L_{d}$ in the descriptor model. Just as an example, the Scharber model suggests that copper phthalocyanine (CuPc) is better than the parent (2,4-bis[4-( $\mathrm{N}, \mathrm{N}$-diisobutylamino)-2,6-dihydroxyphenyl] squaraine) (SQ) donor. However, by including $a(E)$, the improved model shows that SQ should be more efficient than CuPC if the film thickness is $<100 \mathrm{~nm}$, which is within the normal range of OPV donor thickness. ${ }^{19,20}$

\section{THE PROPOSED DESCRIPTOR MODEL}

As known, photovoltaics efficiency $(\eta)$ is commonly expressed as

$$
\eta=\frac{V_{\mathrm{oc}} J_{\mathrm{sc}} F F}{P_{\mathrm{in}}}
$$

where $P_{\text {in }}$ is the input power density. Certainly, many factors contribute to $V_{\mathrm{oc}}, J_{\mathrm{sc}}$ and $F F$. The main factors are materials related. Yet, the device design, fabrication quality and operational conditions have major roles as well. For materials screening, it is reasonable to assume that the device design and quality are optimised. So, the merit of the material's potential for photovoltaic depends mainly on its optoelectronic properties. In this section, relations are proposed to link the materials properties to practical estimations for the maximum obtainable values for $V_{\mathrm{oc}} J_{\mathrm{sc}}$ and FF and hence the efficiency.

For OPV technology, the most efficient cells are bulk heterojunction (BHJ) devices. Conceptually, for materials screening, $\mathrm{BHJ}$ device requires multi-purpose multi-dimensional screening; i.e., a matrix of possible devices need to be screened based on a set of acceptors and a set of donors that fulfil the requirements to make a working solar cell. If the sets are small, then, the twodimensional screening is possible. Otherwise, it can become intractable. So, most of the related large-scale screening is performed for single-layer OPV. ${ }^{12,13,18}$ However, as BHJ devices allow for greater thickness than single-layer ones, the assumed thickness should be larger than the actual 'exciton' diffusion length $\left(L_{x d}\right)$, which is usually $\ll 100 \mathrm{~nm} .{ }^{19-21}$ In this work, we follow the same track of materials screening for single-layer OPV, but assume that the thickness is equal to the nominal average of $\mathrm{BHJ}$ devices, i.e., around $100 \mathrm{~nm} .^{19,20}$ For practical reasons, the focus is on finding a small set of promising donor materials, for which it will be later possible to find matching acceptors.

The proposed descriptor model parameters will be based on the best experimentally reported efficiencies for different organic, inorganic and organometallic materials. These data are tabulated in Tables 1-3. There are some better reported efficiencies; unfortunately, there are no details about the performance parameters of these cells. So, we limit the analysis to the best reported cells with full details. The used reference materials are:

Excitonic:SQ, DTS (5,5-bis(4-(7-hexylthiophen-2-yl)thiophen-2yl)-[1,2,5]thiadiazolo[3,4-c]pyridine-3,3-di-2-ethylhexylsilylene2,2'-bithiophene), CuPc, ZnPc (zinc phthalocyanine), DBP ((dibenzo([f,f]-4,4',7,7'-tetraphenyl)diindeno[1,2,3-cd:1', $\left.2^{\prime}, 3^{\prime}-\mathrm{Im}\right]$ perylene), P3HT (poly(3-hexylthiophene-2,5-diyl), PTB7 (poly[ [4,8-
Table 1. The efficiencies and cell performances for the most-efficient reported inorganic homojunction solar cells with full data

\begin{tabular}{lrrrcccc}
\hline & $\mathrm{E}_{g}(\mathrm{eV})$ & $\mathrm{L}(\mu \mathrm{m})$ & $\mathrm{V}_{o c}(\mathrm{~V})$ & $\mathrm{J}_{s c}\left(\mathrm{~mA} / \mathrm{cm}^{2}\right)$ & $F F$ & $\eta$ & Reference \\
\hline $\mathrm{Si}$ & 1.12 & 200 & 0.74 & 41.8 & 82.7 & 25.6 & 63 \\
$\mathrm{GaAs}$ & 1.43 & 1 & 1.122 & 29.68 & 86.5 & 28.8 & 64 \\
$\mathrm{InP}$ & 1.35 & 3 & 0.878 & 29.5 & 85.4 & 22.1 & 65 \\
\hline
\end{tabular}

Abbreviation: $\mathrm{FF}$, fill factor.

Table 2. The efficiencies and cell performances for the most-efficient reported inorganic and hybrid heterojunction junction solar cells with full data

\begin{tabular}{lccccccc}
\hline & $\mathrm{E}_{g}(\mathrm{eV})$ & $\mathrm{L}(\mu \mathrm{m})$ & $\mathrm{V}_{\text {oc }}(\mathrm{V})$ & $\mathrm{J}_{s c}\left(\mathrm{~mA} / \mathrm{cm}^{2}\right)$ & $F F$ & $\eta$ & Reference \\
\hline GaInP & 1.81 & 1 & 1.455 & 16.04 & 89.3 & 20.8 & 66 \\
CdTe & 1.45 & 3 & 0.872 & 29.47 & 79.5 & 20.4 & 67 \\
$\mathrm{CIGS}$ & 1.21 & 2 & 0.752 & 35.3 & 77.2 & 20.5 & 68 \\
$\mathrm{MAPb}_{3}$ & 1.42 & 0.3 & 1.07 & 21.5 & 67.0 & 15.4 & 69 \\
\hline
\end{tabular}

Abbreviations: CIGS, Culn $\mathrm{Ga}_{(1-x)} \mathrm{Se}_{2} ; \mathrm{FF}$, fill factor; $\mathrm{MAPbl}_{3},\left(\mathrm{CH}_{3} \mathrm{NH}_{3}\right) \mathrm{Pbl}_{3}$.

Table 3. The efficiencies and cell performances for some of the mostefficient reported organic solar cells with full data

\begin{tabular}{lcccccc}
\hline & $\mathrm{E}_{g}(\mathrm{eV})$ & $\mathrm{V}_{o c}(\mathrm{~V})$ & $\mathrm{J}_{s c}\left(\mathrm{~mA} / \mathrm{cm}^{2}\right)$ & $F F$ & $\eta$ & Reference \\
\hline SQ & 1.47 & 0.86 & 13.6 & 52 & 6.1 & 70 \\
DTS & 1.40 & 0.78 & 14.4 & 59.3 & 6.7 & 71 \\
CuPc & 1.46 & 0.54 & 15 & 61 & 5.4 & 72 \\
ZnPc & 1.39 & 0.66 & 12 & 64 & 5.0 & 73 \\
DBP & 1.63 & 1.93 & 13.2 & 66 & 8.1 & 74 \\
P3HT & 1.77 & 0.87 & 11.35 & 75 & 7.4 & 75 \\
PTB7 & 1.51 & 0.75 & 17.46 & 70 & 9.2 & 57 \\
\hline
\end{tabular}

Abbreviations: CuPc, copper phthalocyanine; DTS, (5,5-bis(4-(7-hexylthiophen-2-yl)thiophen-2-yl)-[1,2,5]thiadiazolo[3,4-c]pyridine-3,3-di-2-ethylhexylsilylene-2,2'-bithiophene); FF, fill factor; PTB7, (poly[ [4,8-bis[(2-ethylhexyl) oxy]benzo[1,2-b:4,5-b0]dithiophene-2,6-diyl][3-fluoro-2-[(2-ethylhexyl)carbonyl]thieno[3,4-b]thiophenediyl]]); $\mathrm{P} 3 \mathrm{HT}, \quad$ (poly(3-hexylthiophene-2,5diyl); SQ, (2,4-bis[4-(N,N-diisobutylamino)-2,6-dihydroxyphenyl]squaraine); $\mathrm{ZnPc}$, zinc phthalocyanine.

bis[(2-ethylhexyl)oxy]benzo[1,2-b:4,5-b0]dithiophene-2,6-diyl][3fluoro-2-[(2-ethylhexyl)carbonyl]thieno[3,4-b]thiophenediyl]]), Non-excitonics:Si, GaAs, InP, GalnP, CdTe, Culn $\mathrm{Ga}_{(1-x)} \mathrm{Se}_{2}$ (CIGS) and $\left(\mathrm{CH}_{3} \mathrm{NH}_{3}\right) \mathrm{Pbl}_{3}\left(\mathrm{MAPbl}_{3}\right)$.

Before presenting the descriptor model, it is useful to discuss the solar photon flux density $\left(\phi_{\mathrm{ph}}\right)$ and to introduce simple approximations for the maximum obtainable current density $\left(J_{\mathrm{ph}}\right)$ The reference density is tabulated by the American Society for Testing and Materials standard (ASTM G173-03) for AM0, AM1.5g and $A M 1.5 \mathrm{~d} .^{22}$ For flat panels, $A M 1.5 \mathrm{~g}$ is more appropriate and it will be used in this paper. If all the photons above a given $E_{\mathrm{g}}$ are absorbed (i.e., the reflection is neglected and the thickness of the absorbing material is large enough) and each photon was to generate one exciton, the maximal photogenerated current $J_{\text {ph }}$ is

$$
J_{\mathrm{ph}}=q \int_{E \mathrm{~g}}^{\infty} \phi_{\mathrm{ph}}(E) \mathrm{d} E,
$$

where $q$ is the electron charge. $J_{\mathrm{ph}}$ for the different solar spectra is shown in Figure 1. In the Scharber model, $J_{\mathrm{sc}}$ is assumed to be a fraction of $J_{\text {ph }}$ associated with AM1.5g spectrum. 
As $J_{\mathrm{ph}}$ is used routinely in solar cells calculations, it would be useful to approximate $J_{\mathrm{ph}}$ as a function of $E_{\mathrm{g}}$ in the target range between 1 and $2 \mathrm{eV}$. This will be used later to develop the improved model. Using the data shown in Figure 1, three possible expressions for approximating $J_{\mathrm{ph}}$ as a function of $E_{\mathrm{g}}$ can be suggested:

$$
\begin{aligned}
& \tilde{J}_{\mathrm{ph}, 1}=a_{1} \exp \left(-b_{1} E_{\mathrm{g}}\right), \\
& \tilde{J}_{\mathrm{ph}, 2}=A-\delta J E_{\mathrm{g}}+a_{2} \exp \left(-b_{2} E_{\mathrm{g}}\right)
\end{aligned}
$$

and

$$
\tilde{J}_{\mathrm{ph}, 3}=a_{3} \exp \left(-b_{3} E_{\mathrm{g}}^{\mathrm{c}_{3}}\right) \text {. }
$$

The parameters resulting in the best fit (Figure 2) are:

- for $\tilde{J}_{\mathrm{ph}, 1}, a_{1}=123.62$ and $b_{1}=1.0219$,

- for $\tilde{J}_{\mathrm{ph}, 2}, a_{2}=0.09097, b_{2}=-2.14, A=85.02$ and $\delta J=38.69$,

- for $\tilde{J}_{\mathrm{ph}, 3}, a_{3}=73.531, b_{3}=0.440$ and $c_{3}=1.8617$.

For its simplicity and good accuracy, the expression for $\tilde{J}_{\mathrm{ph}, 3}$ will be used in this work. It will be referred to as $\tilde{J}_{\mathrm{ph}}$, and, correspondingly, the numerical index will be dropped also for the fitting parameters $a, b$ and $c$.

Parameters $V_{\mathrm{oc}}, J_{\mathrm{sc}}$ and FF are tightly coupled. Thus, to have estimations using only $E_{\mathrm{g}}, a(E)$, and $L_{\mathrm{d}}$, many approximations are

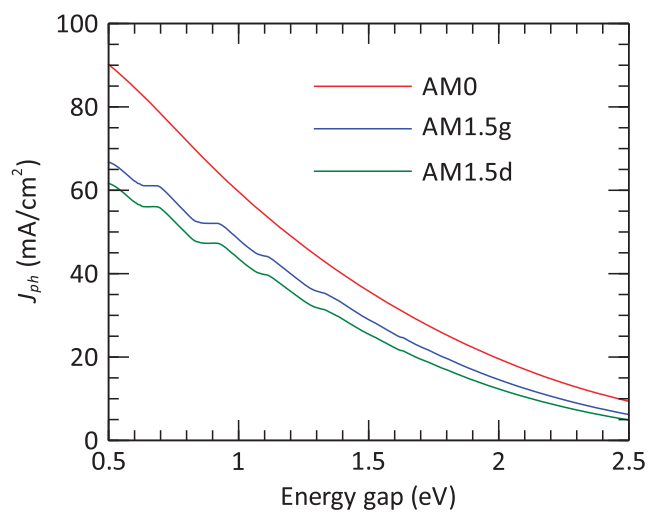

Figure 1. J $J_{\mathrm{ph}}$ versus $E_{\mathrm{g}}$ corresponding to $\mathrm{AMO}, \mathrm{AM} 1.5 \mathrm{~g}$ and $\mathrm{AM} 1.5 \mathrm{~d}$ spectra.

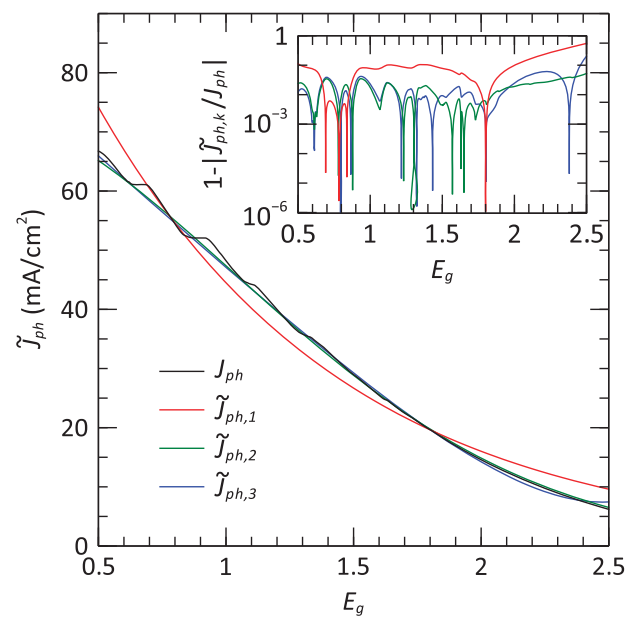

Figure 2. The approximated $J_{\mathrm{ph}}$ versus $E_{\mathrm{g}}$. In the inset, the errors are plotted versus $E_{\mathrm{g}}$ in logarithmic scale. needed. In this work, we will start by estimating $V_{\text {oc }}$ as a function of $E_{\mathrm{g}}$, where the extracted current is assumed as a fraction of $J_{\mathrm{ph}}$. Then, $E_{\mathrm{g}}, a(E)$, and $L_{\mathrm{d}}$ will be used to estimate $J_{\mathrm{sc}}$. Finally, $F F$ is estimated based on $V_{\text {oc }}$.

The open-circuit voltage $\left(V_{\text {oc }}\right)$

Theoretically, $V_{\text {oc }}$ is the maximum voltage that a solar cell can apply to an external load. It is essentially the difference between electron and hole quasi-Fermi levels resulted from photoexcitation. Typically, it is assumed to be upper bounded by $E_{\mathrm{g}} / q$, which is standardly defined-unlike the Scharber model-as the difference between highest occupied molecular orbital and lowest unoccupied molecular orbital of a single absorbing material. In the highly unlikely case of extreme charge accumulation, it can exceed the gap. Many relations between $V_{\text {oc }}$ and $E_{\mathrm{g}}$ were suggested. $^{8-10,23-25}$ Almost all of them are based on the Shockley diode equation (assumed ideal with the identity number set to unity) when the net current vanish. This leads to

$$
V_{\mathrm{oc}}=\frac{k_{\mathrm{B}} T}{q} \ln \left(\frac{J_{\mathrm{sc}}}{J_{0}}+1\right),
$$

where $k_{\mathrm{B}}$ is the Boltzmann constant, $T$ is the cell temperature and $J_{0}$ is the reverse saturation current density. The differences between the suggested models are due to the different assumptions for $J_{\mathrm{sc}}$ and $J_{0}$. For $J_{\mathrm{sc}}$ in this subsection, it is assumed to be a fraction of $J_{\mathrm{ph}}$. This is acceptable as the scaling constant will be considered by the fitting parameters. As for $J_{0}$, many models and empirical equations were suggested. ${ }^{26-29}$ Among the best approximations is the Wanlass equation, ${ }^{28}$ where the values of $J_{0}$ of many of the commonly used semiconductors are fitted to very high accuracy. According to his model,

$$
J_{0}=\beta\left(E_{\mathrm{g}}\right) T^{3} \exp \left(-\frac{E_{\mathrm{g}}}{k_{\mathrm{B}} T}\right),
$$

where

$$
\beta\left(E_{\mathrm{g}}\right)=0.3165 \exp \left(2.192 E_{\mathrm{g}}\right) .
$$

in $\mathrm{mA} / \mathrm{cm}^{2} \mathrm{~K}^{3}$. Theoretically, $\beta$ should be constant. However, $E_{\mathrm{g}}$ dependence is introduced empirically as a correction for

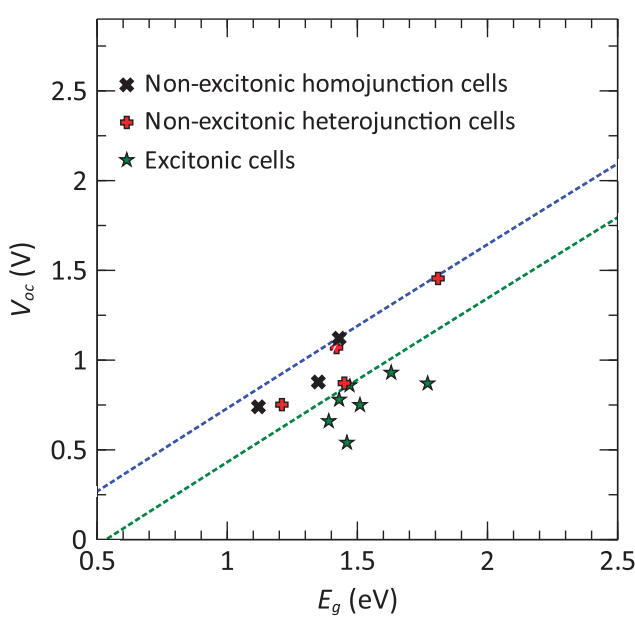

Figure 3. The obtained $V_{\mathrm{oc}}$ versus $E_{\mathrm{g}}$ for the reference materials. The black marks are for inorganic homojunction cells (non-excitonic), the red marks are for the inorganic and organometallic heterojunction junction solar cells (non-excitonic), and the green ones are for the organic cells (excitonic). The two dotted lines are for different values of $V_{\mathrm{L}}$. The blue line is for $V_{\mathrm{L} 0}=0.2 \mathrm{~V}$, which fits non-excitonic cells, and the green line is for $V_{\mathrm{L} 0}=0.5 \mathrm{~V}$ for excitonic cells. 
homojunction 'solar cells' operation. ${ }^{28-30}$ The same form was suggested also for OPVs, which are heterojunction devices, but with slightly smaller value for the prefactor. ${ }^{31,32}$ So, by applying a fraction of $\tilde{J}_{\mathrm{ph}}$ (Equation 5) and $J_{0}$ in equation (6) and by considering the fact that $J_{0}$ is very small quantity, we obtain

$$
\tilde{V}_{\text {oc }}=E_{\mathrm{g}}-\tilde{V}_{\mathrm{L}}
$$

where at room temperature (at $300 \mathrm{~K}$ )

$$
\tilde{V}_{\mathrm{L}}=0.0114 E_{\mathrm{g}}^{1.8617}+0.057 E_{\mathrm{g}}+V_{\mathrm{L} 0}
$$

and $V_{\mathrm{LO}}$ is used as a fitting parameter (thus the differences in the prefactors of $J_{0}$ are accounted for).

In homojunction solar cells, the losses are mainly due to the materials and the excitation; i.e., the losses due to the device design are-in principle-avoidable. For heterojunction devices, the energy offsets between the layers add to the voltage loss. For non-excitonic solar cells, the binding energy of exciton is small and hence it can be dissociated thermally or by a potential gradient. As a result, the heterojunction offset can be made small. On the other hand, in excitonic cells, the binding energy is large and the band offset is used to dissociate excitons. Thus, a considerable additional loss in the voltage is unavoidable. Therefore and as aforementioned, it is essential to make distinction between the two classes of excitonic and nonexcitonic cells. The original Scharber model considers the

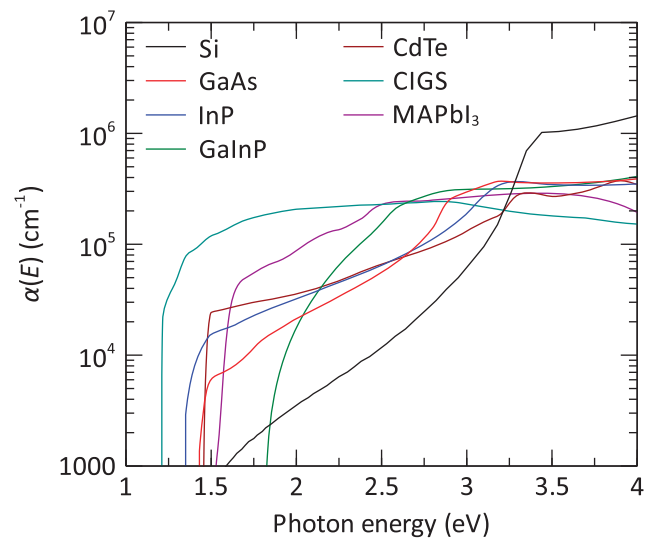

reduction due to band offset. However, this is routinely ignored in materials screening as it adds extra constraint on the acceptor.

Figure 3 maps the obtained $V_{\text {oc }}$ to $E_{\mathrm{g}}$ for the reference materials. Clearly for non-excitonic cells, the blue line $\left(V_{\mathrm{LO}}=0.2 \mathrm{~V}\right)$ line provides a good estimation for the upper limit. On the other hand, the maximum experimentally measured $V_{\text {oc }}$ values for excitonic cells are much lower. This is mainly due to the sizable, yet needed heterojunction band offset. Thus, larger $V_{\mathrm{LO}}$ is indispensable. Here this parameter is set to the lowest reported voltage loss in organic cells. As can be seen in Table 3, it is $0.61 \mathrm{~V}$ for SQ-based solar cell leading to $V_{\mathrm{L} 0} \approx 0.5 \mathrm{~V}$.

The short-circuit current $\left(J_{\mathrm{sc}}\right)$

As stated in the introduction, in the Scharber model, $J_{\mathrm{sc}}$ is assumed to amount to a constant fraction of $J_{\mathrm{ph}}$. Usually, 0.65 is used as the scaling parameter. So,

$$
\tilde{J}_{\mathrm{sc}, \mathrm{Sch}}=0.65 J_{\mathrm{ph}} \text {. }
$$

The two most crucial deficiencies of the Scharber model, namely, the assumption of a homogeneous absorption spectrum (above the bandgap) and that the transport is very efficient such that $L_{d}$ is much larger than the absorption length, can be addressed by explicitly considering the spectral inhomogeneity (using $a(E)$ ) and by introducing a proper characterisation of $L_{d}$. This shall result in improved predictions while not over-complicating the descriptor model.

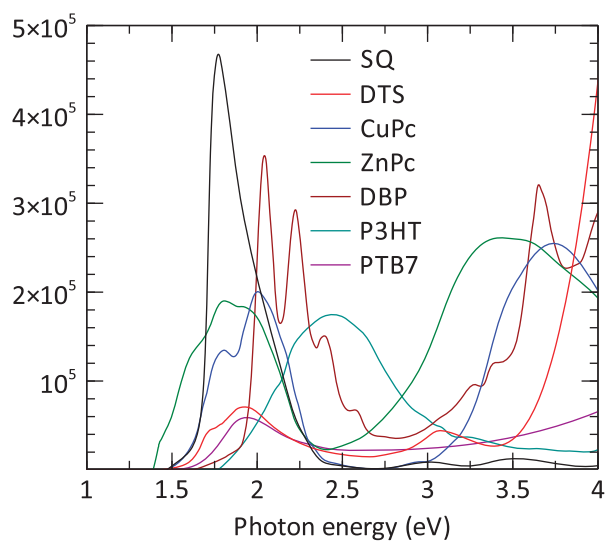

Figure 4. The absorption coefficients of the reference materials; left: non-excitonic cells, right: excitonic cells. The data are extracted from various sources. ${ }^{49-56}$
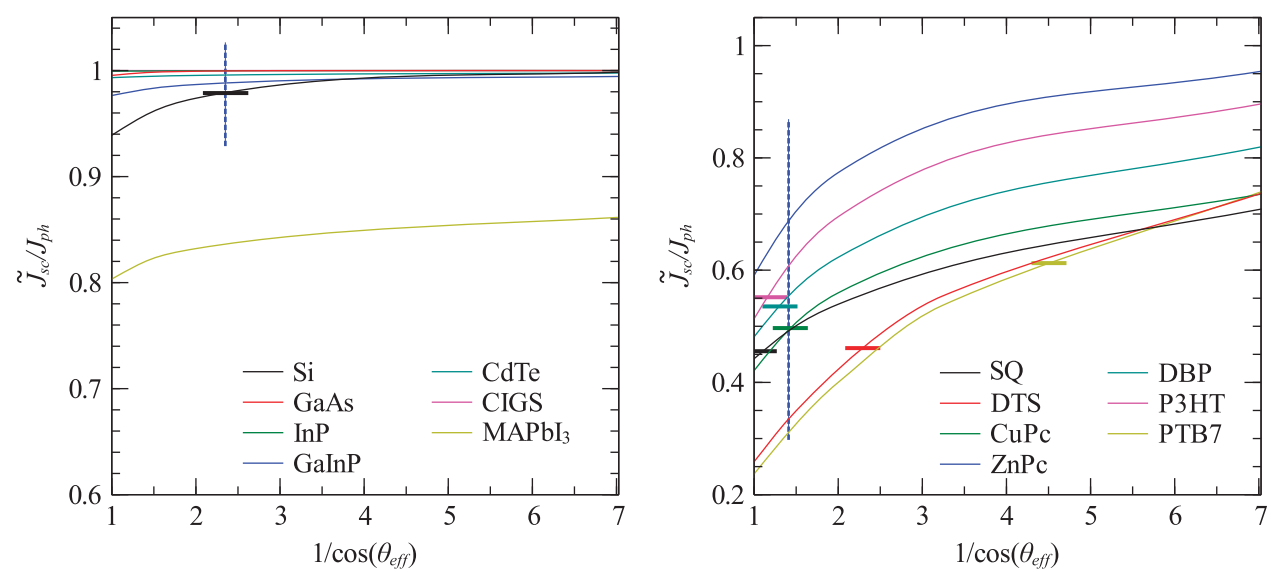

Figure 5. The ratio between $\tilde{J}_{\mathrm{sc}}$ and $J_{\mathrm{ph}}$ for the reference materials at different $1 / \cos (\theta)$; left: non-excitonic cells, right: excitonic cells. The small marks correspond to the maximum reported ratios. For the materials without shown marks, the ratios match the maximum possible values without scattering. This is mainly due to their strong absorptions and very efficient transports. 
The absorption spectrum $a(E)$ can be computed by means of electronic structure calculations, often based on semiclassical approaches. These calculations also provide numerical values for $E_{\mathrm{g}}$. So, there is no additional atomistic calculation needed for $a(E)$. However, the calculation of $a(E)$ from these common inputs can be computationally expensive. This fact shall be considered during the design of high-throughput screening.

Commonly, $a(E)$ is calculated by semiclassical approach where the electrons are treated quantum mechanically through the electronic structure and the field is treated classically. The details vary based on the used method for electronic structure calculations. From electronic structure calculations, the complex dielectric function $\left(\varepsilon(E)=\varepsilon_{1}(E)+i \varepsilon_{2}(E)\right)$ can be calculated. $\varepsilon_{2}(E)$ is calculated by considering all the possible transitions from occupied to unoccupied states. For each transition, its contribution into $\varepsilon_{2}(E)$ is proportional to the square of the matrix element. Then, $\varepsilon_{1}(E)$ is calculated from $\varepsilon_{2}(E)$ using the Kramers-Kronig transformation. Finally, both the refractive index $n(E)$ and $a(E)$ are calculated from the relation $\sqrt{\varepsilon_{1}(E)+i \varepsilon_{2}(E)}=n(E)+i a(E) \hbar c / q E$, where $\hbar$ is the Planck constant and $c$ is the speed of light.

As aforementioned, the transport is commonly characterised by $L_{d}$. Calculating $L_{d}$ requires very time-consuming computing, which will complicate the materials screening process. To avoid that, each material is given a value of $L_{d}$, which is characteristic for the family of compounds it belongs to.

Many parameters govern $L_{d}$. Some of them are related to the intrinsic properties of materials and many others are due to the fabrication quality. For non-excitonic cells, the minority carrier

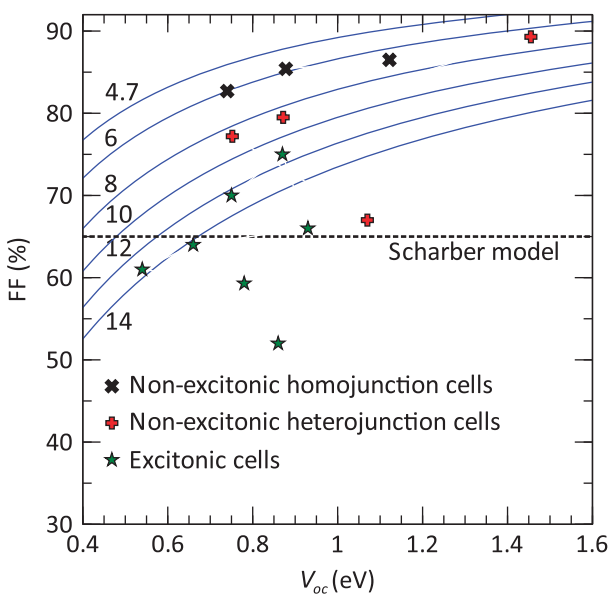

Figure 6. The obtained $F F$ versus $V_{o c}$ for the reference materials. The black marks are for inorganic homojunction cells (non-excitonic), the red marks are for the inorganic and hybrid heterojunction solar cells (non-excitonic) and the green ones are for the organic cells (excitonic). The solid lines are for six different values of $a$, whereas the dotted black line is the value suggested by Scharber model (Table 4). diffusion is the main process and it is limited mainly by material growth quality. For defect-free indirect bandgap materials, lifetimes are in milliseconds and the mobilities are high, which give rise of few hundred microns to $L_{\mathrm{d}} \cdot{ }^{33,34}$ However, for direct gap materials, the lifetime is significantly reduced because of the band-to-band recombination. Thus, $L_{d}$ is reduced to a range between few microns and few tens of microns. ${ }^{35-37}$ As for organometallic materials, $L_{d}$ is estimated to be $>1 \mu \mathrm{m}$ for methylammonium lead iodide. ${ }^{38}$

On the other hand, the main limiting factor in excitonic solar cells is the exciton diffusion length. ${ }^{39,40}$ The exciton cannot dissociate at the same location at which it was generated. Rather, it has to travel by hopping to the nearest interface to dissociate. Thus, the transport is limited by exciton diffusion rather than the free carrier diffusion. Exciton diffusion length in organic solar cell materials is normally $<0.1 \mu \mathrm{m} .{ }^{19-21}$ In this work, we assume the following values for $L_{d}$ as characteristic for the following material families:

- for indirect-gap semiconductors, $L_{\mathrm{d}} \approx 200 \mu \mathrm{m}$,

- for direct-gap semiconductors, $L_{\mathrm{d}} \approx 10 \mu \mathrm{m}$,

- for organometallic semiconductors, $L_{\mathrm{d}} \approx 0.6 \mu \mathrm{m}$,

- for excitonic cells, $L_{d} \approx 0.1 \mu \mathrm{m}$.

Conceptually, $J_{\mathrm{sc}}$ is the difference between photogenerated and recombination currents, i.e.,

$$
J_{\mathrm{sc}}=J_{\mathrm{g}}-J_{\mathrm{r}}
$$

In an ideal situation, it is assumed that the thickness of the absorber layer is so large that all photons above $E_{\mathrm{g}}$ are absorbed. Practically, the carrier collection and hence the absorber layer thickness are limited by $L_{d}$. Therefore, the maximum possible photogenerated current is ${ }^{41-44}$

$$
J_{\mathrm{g}}=q \int_{E \mathrm{~g}}^{\infty} \int_{-\pi / 2}^{\pi / 2} \phi_{\mathrm{ph}}(E) P\left(\theta, \theta_{\mathrm{inc}}, E\right)\left[1-e^{-a(E) L_{\mathrm{d}} / \cos (\theta)}\right] \mathrm{d} \theta \mathrm{d} E
$$

where $P\left(\theta, \theta_{\text {inc }}, E\right)$ is an angular distribution function that accounts for the scattering of the light at angle $\theta$ in the absorbing layer depending on the incidence angle $\theta_{\text {inc }}$ and photon energy. The scattering results in increasing-positively-the optical path of the light in the absorbing layer by a factor of $1 / \cos (\theta)$. From a device-performance perspective, it is important to have $L_{d}$ much larger than the absorption length $\left(L_{\alpha} \propto 1 / a(E)\right)$. If $L_{d} \gg L_{a}$, the second term in the square bracket gets diminished and $J_{g}$ increases. Otherwise, $J_{\mathrm{g}}$ is reduced considerably.

Obviously, $P\left(\theta, \theta_{\text {inc }}, E\right)$ depends on many factors mainly related to the films morphology and microstructure and the structure of interfaces. For the modelling of the distribution function, many different distributions were suggested. ${ }^{42,43,45-48}$ In this work, to keep the model simple, we can combine the scattering effects in a

Table 4. A summary of the original Scharber model and proposed model for both excitonic and non-excitonic solar cells. For the original Scharber model, $\Delta V$ is the band offset

\begin{tabular}{llll}
\hline & $\begin{array}{l}\text { The original } \\
\text { model }\end{array}$ & $\begin{array}{c}\text { The proposed model } \\
\text { for excitonic cells }\end{array}$ & $\begin{array}{c}\text { The proposed model } \\
\text { for non-excitonic cells }\end{array}$ \\
\hline$V_{\mathrm{oc}}$ & $E_{\mathrm{g}}-0.3-\Delta V$ & $E_{\mathrm{g}}-0.5-0.0114 E_{\mathrm{g}}^{1.8617}$ & $E_{\mathrm{g}}-0.2-0.0114 E_{\mathrm{g}}^{1.8617}$ \\
$J_{\mathrm{sc}}$ & $0.65 J_{\mathrm{ph}}\left(E_{\mathrm{g}}\right)$ & $-0.057 E_{\mathrm{g}}$ & $-0.057 E_{\mathrm{g}}$ \\
$\mathrm{FF}$ & 0.65 & $\tilde{J}_{\mathrm{g}}\left(E_{\mathrm{g}}, a(E), L_{\mathrm{d}}, \pi / 4\right)$ & $\tilde{J}_{\mathrm{g}}\left(E_{\mathrm{g}}, a(E), L_{\mathrm{d}}, \pi / 2.75\right)$ \\
\hline Abbreviation: $\mathrm{FF}$, fill factor. & & $V_{\mathrm{oc}}\left(V_{\mathrm{oc}}+12 k_{\mathrm{B}} T_{\mathrm{c}}\right)$ & $V_{\mathrm{oc}}\left(V_{\mathrm{oc}}+6 k_{\mathrm{B}} T_{\mathrm{c}}\right)$ \\
\hline
\end{tabular}


single effective angle $\theta_{\text {eff. }}$ So, $J_{\mathrm{g}}$ becomes

$$
\tilde{J}_{\mathrm{g}}\left(E, a(E), L_{\mathrm{d}}, \theta_{\text {eff }}\right)=q \int_{E \mathrm{~g}}^{\infty} \phi_{\mathrm{ph}}(E)\left[1-e^{-a(E) L_{\mathrm{d}} / \cos \left(\theta_{\text {eff }}\right)}\right] \mathrm{d} E
$$

The way $\theta_{\text {eff }}$ is determined will be shown at the end of this subsection.

As for the recombination, there are many mechanisms contributing to it. This is accounted for empirically by $L_{d}$ and it can be adjusted further by a proper fitting of $\theta_{\text {eff. So, }} J_{\text {sc }}$ can be approximated by a similar form of equation (14), but with a slightly different $\theta_{\text {eff, }}$ that will be determined based on the actual performances and absorption spectra of the reference materials.

Figure 4 shows the absorption spectra of the reference materials, which are extracted from various sources. ${ }^{49-56}$ For the known non-excitonic cells, it is evident that due to the extended absorption spectrum above $E_{\mathrm{g}}, a(E)$ is smooth, whereas the organic materials show a strongly fluctuating bands. For example, the absorption of SQ is strong only between 1.5 and $2.3 \mathrm{eV}$. Thus, a large portion of solar radiation is not absorbed due to the fact that the device thickness is small.

To determine $\theta_{\text {eff, }}$ the ratios $\tilde{J}_{\mathrm{sc}} / \tilde{J}_{\mathrm{ph}}$ are calculated and plotted against $1 / \cos \left(\theta_{\text {eff }}\right)$ (Figure 5$)$. For non-excitonic cells, the effect of $\theta_{\text {eff }}$ for most materials is negligible due to their strong absorption and due to the fact that the growth quality of the studied materials is high and hence the assumed $L_{d}$ is large. The exceptions are for Si due to its weak absorption and for $\mathrm{MAPbl}_{3}$ due to its relatively short $L_{\mathrm{d}}$. For $\mathrm{Si}, \theta_{\text {eff }} \approx \pi / 2.75$ is needed to match the obtained $J_{s c}$. So, this value will be used for non-excitonic cells. For excitonic cells, $\theta_{\text {eff }}=\pi / 4$ is a good approximation for most of the studied materials. The exception is for PTB7, where the difference between the reported $J_{\mathrm{sc}}$ and the calculated value from absorption spectrum is high. This can be due to an extremely efficient light trapping used to make the cell. ${ }^{57}$ However, to match most of the reported maximum values, $\theta_{\text {eff }}=\pi / 4$ is suitable and will be used for excitonic cells.

\section{The fill factor}

The third performance parameter is the FF. Practically, many physical mechanisms contribute to it and consequently many models have been suggested to estimate it. ${ }^{5-61}$ Generally, the suggested models are based on the relationship between current and voltage; but with different assumptions on the causes and values of shunt and series resistances. FF is usually represented as a function of $V_{\text {oc }}$, which depends as shown above on $E_{\mathrm{g}}$.

One of the simplest-yet reasonably accurate-forms suggested by Green ${ }^{61}$ for conventional inorganic semiconductors is

$$
F F=\frac{V_{\text {oc }}}{V_{\text {oc }}+a k_{\mathrm{B}} T}
$$

Originally, he suggested $a=4.7$. However, this factor can be adjusted for other solar cell technologies. On the basis of the best reported cells, $a=6$ and $a=12$ fit better the upper limits of the measured FF for non-excitonic and excitonic solar cells, respectively, as shown in Figure 6, where $T$ is the room temperature.

\section{MODEL IMPLEMENTATION AND EVALUATION}

The parameterized expressions used for the three performance factors $\left(V_{\mathrm{oc}}, J_{\mathrm{sc}}\right.$ and $\left.F F\right)$ in the original Scharber model and the presented descriptor are summarised in Table 4.

In the first analysis, we compare the predictions of the two models for excitonic materials with the available experimental data (Table 3). For the original Scharber model, three reasonable values $^{12,14,62}$ for $\Delta V$ are assumed in the analysis; namely, $0.2,0.3$ and $0.4 \mathrm{~V}$. The results are shown in Figure 7. As can be observed, the improved model outperforms considerably the original Scharber model in the estimations of $J_{\mathrm{sc}}$ except for PTB7. For $V_{\text {oc }}$ the presented model provides good estimation for most of the studied materials. As for the original Scharber model, this depends obviously on $\Delta V$. For $\eta$, the presented model outperforms in most cases the original one. The original model performs
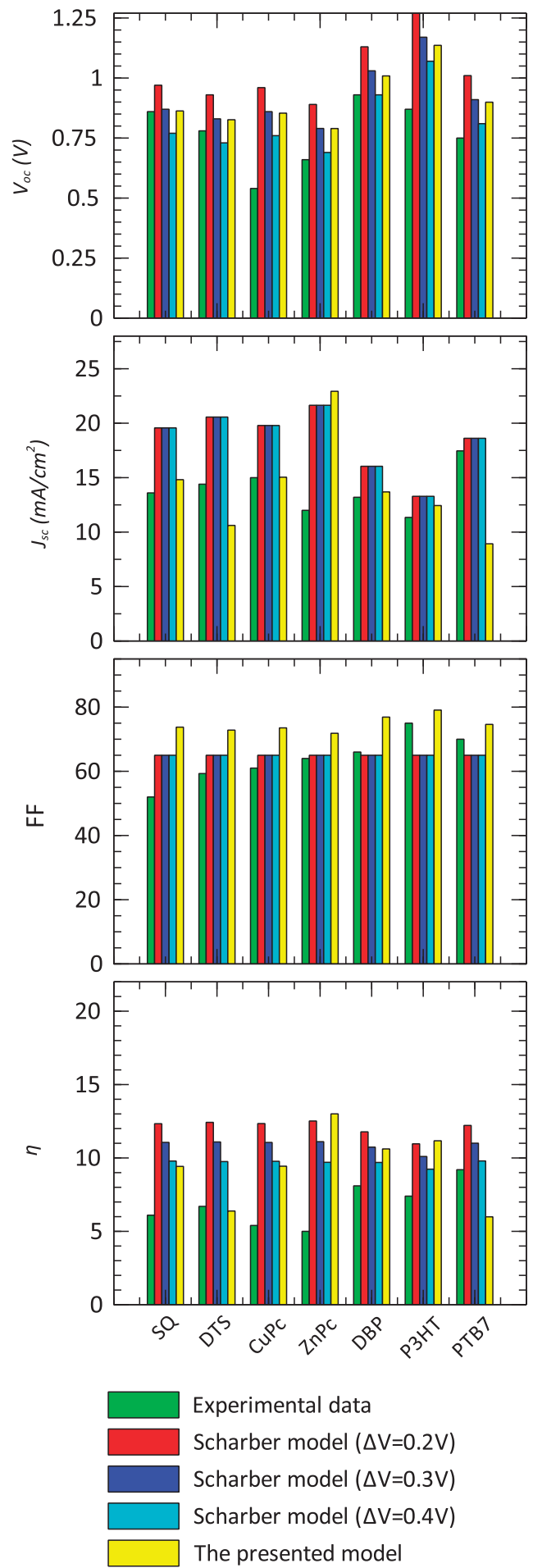

Figure 7. Comparison for organic (excitonic) solar cell performances of the relevant reference materials as estimated by the presented and the Scharber (for three different values for $\Delta V$ ) models, and as experimentally published for the best reported cells. 


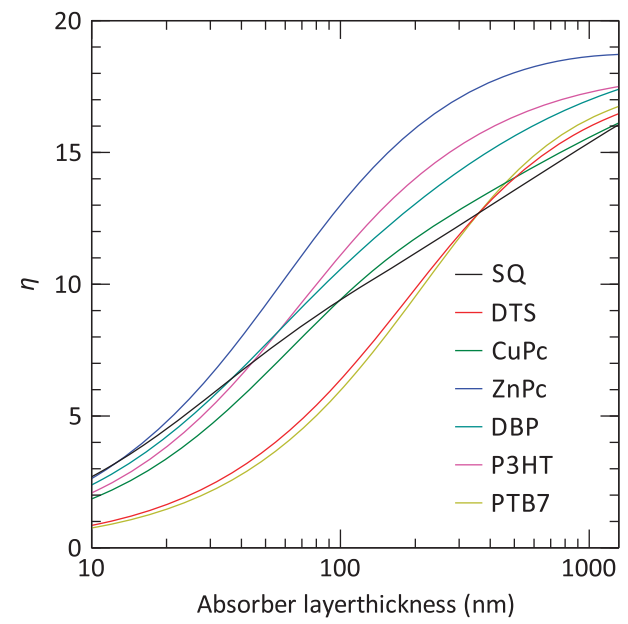

Figure 8. The estimated solar cell efficiencies for the reference organic (excitonic) materials as a function of the absorbing layer thickness.

generally better only in the estimation of FF. However, FF depends extremely on the device design and optimisation, unlike other parameters that are mostly materials dependent. The improved model suggests that the obtained $F F$ values are smaller than the predictions. Thus, there is a reasonable room for improvement through device optimisation.

To illustrate the importance of considering $L_{d}$, the presented method is used to estimate conversion efficiencies of the array of organic materials studied as a function of thicknesss as shown in Figure 8. Clearly, material potential ranking varies with the thickness. For example, for very thin films $(L<50 \mathrm{~nm})$, SQ is predicted to show better efficiency when compared with all other materials, except ZnPc. Also, PTB7- and DTS-based solar cells would result in better efficiencies when compared with CuPc and SQ only for relatively thicker films.

In the next analysis, the improved model is applied to the reference non-excitonic materials. As aforementioned, the original Scharber model was designed for OPVs. It can be adjusted to also work for inorganic cells in a similar way as our proposed model, i.e., by working with two sets of parameters. Figure 9 shows a comparison between the reported experimental efficiencies and the estimated ones by the proposed model for non-excitonic cells. Again, the model provides very good estimations. For the welloptimised devices such as $\mathrm{Si}$ and $\mathrm{GaAs}$, the presented model suggests that the room of improvement is limited. However, it indicates that there is a possibility to considerably improve the performance of $\mathrm{MAPbl}_{3}, \mathrm{CIGS}, \mathrm{CdTe}$ and InP.

The last analysis is for the effect the absorber layer thickness on the expected efficiencies for the non-excitonic solar cell materials. The results are shown in Figure 10. The expected efficiencies for all the studied materials saturate after few micrometre except for Si solar cells. It takes very thick layer to reach a reasonable efficiency. As known, this is due to its weak absorption. ${ }^{4,5,47,63}$

\section{CONCLUSION}

A descriptor model for solar cell efficiencies estimation is developed. Relative to the original Scharber model, the developed model presented here revisits the three main performance factors $\left(V_{\mathrm{oc}}, J_{\mathrm{sc}}\right.$ and $F F$; Equation 1$)$. For the short-circuit current $\left(J_{\mathrm{sc}}\right)$, the model takes in full account the details of the absorption spectrum $a(E)$ to evaluate the photogenerated current $\left(J_{\mathrm{g}}\right)$, and uses new and more elaborate parametrization for the other components contributing to this quantity, i.e., the scattering distribution and
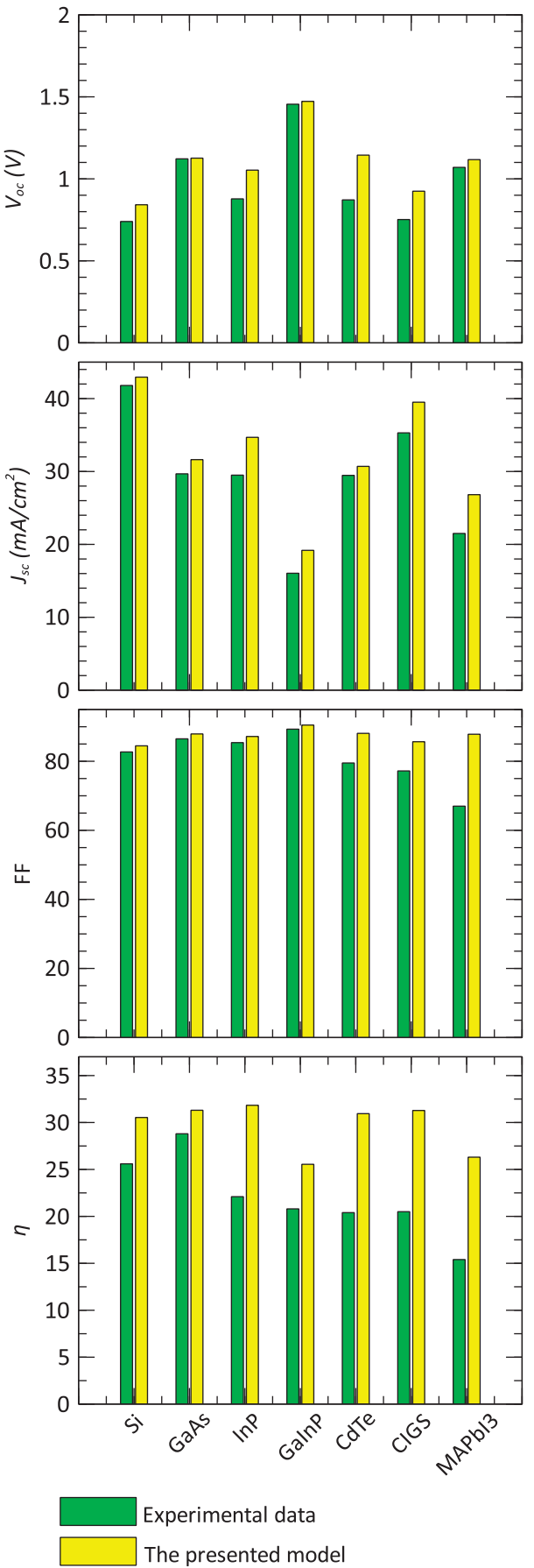

Figure 9. Comparison for non-excitonic solar cell performances of the relevant reference materials as estimated by the presented and as experimental published for the best reported cells.

the diffusion length, which characterize the transport and the recombination. The open-current voltage $V_{\text {oc }}$ is expressed in terms of a power series of the energy gap fitted against available experimental data. The FF is estimated using adjusted empirical model originally suggested by M. Green. ${ }^{61}$ Using two different sets of parameters, the model can be used for both excitonic and nonexcitonic materials.

The analysis of the new model shows that its much better performance arises from the improved predictions for the opencircuit voltage and the short-circuit current $\left(V_{\text {oc }}\right.$ and $\left.J_{\text {sc }}\right)$. On the other hand, the estimation of the original Scharber model for the 


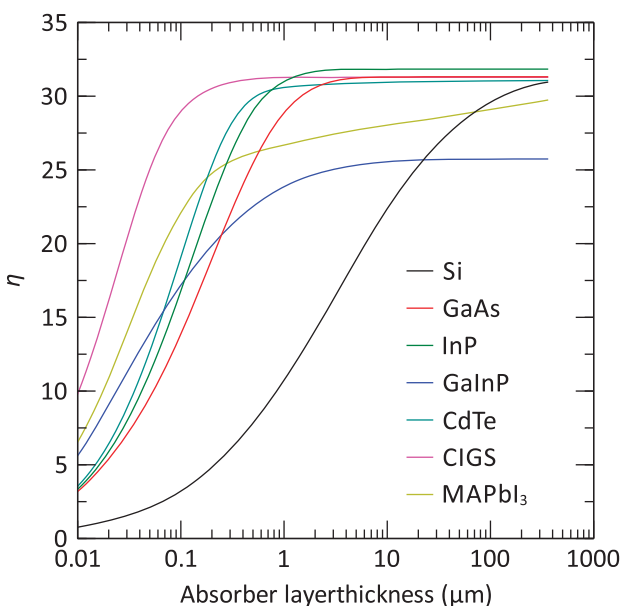

Figure 10. The estimated non-excitonic solar cell efficiencies for the relevant reference materials as a function of the absorbing layer thickness.

FF is slightly better than our model when compared with the experimentally reported values. However, as FF depends extremely on the device design and optimisation, the larger values estimated by the proposed model indicate that the performance of the reference cells can be improved by proper device optimisation.

We expect the proposed descriptor model to allow for more accurate assessments of the performance of light harvesting materials. Even though the results of material screening efforts based on this model are still missing, the model was already shown to be useful to study the change of performance of a given material with the variation of some device parameter such as the layer thickness.

\section{COMPETING INTERESTS}

The authors declare no conflict of interest.

\section{REFERENCES}

1 Azimi, H., Hou, Y. \& Brabec, C. J. Towards low-cost, environmentally friendly printed chalcopyrite and kesterite solar cells. Energy Environ. Sci. 7, 1829-1849 (2014).

2 Habas, S. E., Platt, H. A., van Hest, M. F. \& Ginley, D. S. Low-cost inorganic solar cells: from ink to printed device. Chem. Rev. 110, 6571-6594 (2010).

3 Wadia, C., Alivisatos, A. P. \& Kammen, D. M. Materials availability expands the opportunity for large-scale photovoltaics deployment. Energy Environ. Sci. 43, 2072-2077 (2009).

4 Alharbi, F. et al. Abundant non-toxic materials for thin film solar cells: alternative to conventional materials. Renew. Energy 36, 2753-2758 (2011).

5 Hossain, M. \& Alharbi, F. Recent advances in alternative material photovoltaics. Mater. Technol. 28, 88-97 (2013).

6 Dimroth, F. et al. Wafer bonded four-junction gainp/gaas//gainasp/gainas concentrator solar cells with $44.7 \%$ efficiency. Prog. Photovoltaics Res. Appl. 22 277-282 (2014).

7 Snaith, H. J. Perovskites: the emergence of a new era for low-cost, high-efficiency solar cells. J. Phys. Chem. Lett. 4, 3623-3630 (2013).

8 Nelson, J., Kirkpatrick, J. \& Ravirajan, P. Factors limiting the efficiency of molecular photovoltaic devices. Phys. Rev. B 69, 035337 (2004).

9 Markvart, T. The thermodynamics of optical étendue. J. Opt. A 10, 015008 (2008).

10 Kirk, A. A discussion of fundamental solar photovoltaic cell physics. Physica B 423, 58-59 (2013).

11 Alharbi, F. H. \& Kais, S. Theoretical limits of photovoltaics efficiency and possible improvements by intuitive approaches learned from photosynthesis and quantum coherence. Renew. Sustainable Energy Rev. 43, 1073-1089 (2015).
12 Hachmann, J. et al. The harvard clean energy project: large-scale computational screening and design of organic photovoltaics on the world community grid. $J$. Phys. Chem. Lett. 2, 2241-2251 (2011).

13 Olivares-Amaya, R. et al. Accelerated computational discovery of highperformance materials for organic photovoltaics by means of cheminformatics. Energy Environ. Sci. 4, 4849-4861 (2011).

14 Hachmann, J. et al. Lead candidates for high-performance organic photovoltaics from high-throughput quantum chemistry-the harvard clean energy project. Energy Environ. Sci. 7, 698-704 (2014).

15 Landis, D. D. et al. The computational materials repository. Comput. Sci. Eng. 14, 51-57 (2012)

16 Castelli, I. E. et al. Computational screening of perovskite metal oxides for optimal solar light capture. Energy Environ. Sci. 5, 5814-5819 (2012).

17 Curtarolo, S. et al. The high-throughput highway to computational materials design. Nat. Mater. 12, 191-201 (2013).

18 Scharber, M. C. et al. Design rules for donors in bulk-heterojunction solar cells towards 10\% energy-conversion efficiency. Adv. Mater. 18, 789-794 (2006).

19 Hoppe, H. \& Sariciftci, N. S. Organic solar cells: An overview. J. Mater. Res. 19, 1924-1945 (2004).

20 Forrest, S. R. The limits to organic photovoltaic cell efficiency. MRS Bull. 30, 28-32 (2005)

21 VajjalaáKesava, S. et al. Direct measurements of exciton diffusion length limitations on organic solar cell performance. Chem. Commun. 48, 5859-5861 (2012).

22 ASTM G173-03: Standard tables for reference solar spectral irradiances: direct normal and hemispherical on $37^{\circ}$ tilted surface. Technical Report. Available at http://www.astm.org/Standards/G173.htm (2008).

23 Abrams, Z. R., Gharghi, M., Niv, A., Gladden, C. \& Zhang, X. Theoretical efficiency of 3rd generation solar cells: comparison between carrier multiplication and down-conversion. Sol. Energy Mater. Sol. Cells 99, 308-315 (2012).

24 Würfel, P., Brown, A., Humphrey, T. \& Green, M. Particle conservation in the hotcarrier solar cell. Prog. Photovoltaics Res. Appl. 13, 277-285 (2005).

25 Baruch, P., De Vos, A., Landsberg, P. \& Parrott, J. On some thermodynamic aspects of photovoltaic solar energy conversion. Sol. Energy Mater. Sol. Cells 36, 201-222 (1995).

26 Fan, J., Tsaur, B. \& Palm, B. In Proc. 16th IEEE Photovoltaic Specialists Conference 692-701 (1982).

27 Nell, M. E. \& Barnett, A. M. The spectral pn junction model for tandem solarcell design. IEEE Trans. Electron Devices 34, 257-266 (1987).

28 Wanlass, M. et al. Practical considerations in tandem cell modeling. Sol. Cells 27, 191-204 (1989).

29 Coutts, T. A review of progress in thermophotovoltaic generation of electricity. Renew. Sustainable Energy Rev. 3, 77-184 (1999).

30 Coutts, T. J., Emery, K. A. \& Scott Ward, J. Modeled performance of polycrystalline thin-film tandem solar cells. Prog. Photovoltaics Res. Appl. 10, 195-203 (2002).

31 Potscavage, W. J. Jr, Yoo, S. \& Kippelen, B. Origin of the open-circuit voltage in multilayer heterojunction organic solar cells. Appl. Phys. Lett. 93, 193308 (2008).

32 Katz, E. et al. Temperature dependence for the photovoltaic device parameters of polymer-fullerene solar cells under operating conditions. J. Appl. Phys. 90, 5343-5350 (2001).

33 Stutenbaeumer, U. \& Lewetegn, E. Comparison of minority carrier diffusion length measurements in silicon solar cells by the photo-induced open-circuit voltage decay (ocvd) with different excitation sources. Renew. Energy 20, 65-74 (2000).

34 Fortini, A., Lande, R., Madelon, R. \& Bauduin, P. Carrier concentration and diffusion length measurements by 8 -mm-microwave magnetophotoreflectivity in germanium and silicon. J. Appl. Phys. 45, 3380-3384 (1974).

35 Leon, R. In Proc. 19th IEEE Photovoltaic Specialists Conference vol. 1, 808-812 (1987).

36 Mataré, H. \& Wolff, G. Concentration enhancement of current density and diffusion length in iii-v ternary compound solar cells. Appl. Phys. A 17, 335-342 (1978).

37 Gautron, J. \& Lemasson, P. Photoelectrochemical determination of minority carrier diffusion length in ii-vi compounds. J. Cryst. Growth 59, 332-337 (1982).

38 Stranks, S. D. et al. Electron-hole diffusion lengths exceeding 1 micrometer in an organometal trihalide perovskite absorber. Science 342, 341-344 (2013).

39 Gregg, B. A. Excitonic solar cells. J. Phys. Chem. B 107, 4688-4698 (2003).

40 Gregg, B. A. The photoconversion mechanism of excitonic solar cells. MRS Bull. $\mathbf{3 0}$, 20-22 (2005).

41 Poruba, A. et al. Optical absorption and light scattering in microcrystalline silicon thin films and solar cells. J. Appl. Phys. 88, 148-160 (2000).

$42 \mathrm{Krc}$, J., Zeman, M., Smole, F. \& Topic, M. Optical modelling of thin-film silicon solar cells deposited on textured substrates. Thin Solid Films 451, 298-302 (2004).

$43 \mathrm{Krc}$, J., Smole, F. \& Topic, M. Analysis of light scattering in amorphous si: H solar cells by a one-dimensional semi-coherent optical model. Prog. Photovoltaics Res. Appl. 11, 15-26 (2003). 
44 Müller, J., Rech, B., Springer, J. \& Vanecek, M. Tco and light trapping in silicon thin film solar cells. Sol. Energy 77, 917-930 (2004).

45 Battaglia, C. et al. Light trapping in solar cells: can periodic beat random? ACS Nano 6, 2790-2797 (2012).

46 Callahan, D. M., Munday, J. N. \& Atwater, H. A. Solar cell light trapping beyond the ray optic limit. Nano Lett. 12, 214-218 (2012).

47 Cho, J.-S. et al. Effect of nanotextured back reflectors on light trapping in flexible silicon thin-film solar cells. Sol. Energy Mater. Sol. Cells 102, 50-57 (2012).

48 Kowalczewski, P., Liscidini, M. \& Andreani, L. C. Light trapping in thin-film solar cells with randomly rough and hybrid textures. Opt. Express 21, A808-A820 (2013).

49 Sze, S. M. \& Ng, K. K. Physics of Semiconductor Devices (John Wiley \& Sons, 2006).

50 Weber, M. J. Handbook of Optical Materials vol. 19 (CRC press, 2002).

51 Theodoropoulou, S., Papadimitriou, D., Anestou, K., Cobet, C. \& Esser, N. Optical properties of cuin ${ }_{1-x} \mathrm{ga}_{x} \mathrm{se} 2$ quaternary alloys for solar-energy conversion. Semicond. Sci. Technol. 24, 015014 (2009)

52 Gamliel, S. \& Etgar, L. Organo-metal perovskite based solar cells: sensitized versus planar architecture. RSC Adv. 4, 29012-29021 (2014).

53 Wei, G. et al. Functionalized squaraine donors for nanocrystalline organic photovoltaics. ACS Nano 6, 972-978 (2011).

54 Duché, D. et al. Optical performance and color investigations of hybrid solar cells based on p3ht: Zno, pcpdtbt: Zno, ptb7: Zno and dts (ptth $\left.)_{2}\right)_{2}$ Zno. Sol. Energy Mater. Sol. Cells 126, 197-204 (2014).

55 Fujishima, D. et al. Organic thin-film solar cell employing a novel electron-donor material. Sol. Energy Mater. Sol. Cells 93, 1029-1032 (2009).

56 Senthilarasu, S. et al. Characterization of zinc phthalocyanine (znpc) for photovoltaic applications. Appl. Phys. A 77, 383-389 (2003).

$57 \mathrm{He}$, Z. et al. Enhanced power-conversion efficiency in polymer solar cells using an inverted device structure. Nat. Photon. 6, 591-595 (2012).

58 Qi, B. \& Wang, J. Fill factor in organic solar cells. Phys. Chem. Chem. Phys. 15, 8972-8982 (2013)

59 Taretto, K., Soldera, M. \& Troviano, M. Accurate explicit equations for the fill facto of real solar cells applications to thin-film solar cells. Prog. Photovoltaics Res. Appl. 21, 1489-1498 (2013).

60 Gupta, D., Mukhopadhyay, S. \& Narayan, K. Fill factor in organic solar cells. Sol. Energy Mater. Sol. Cells 94, 1309-1313 (2010).

61 Green, M. A. Accuracy of analytical expressions for solar cell fill factors. Sol. Cells 7, 337-340 (1982)

62 Dou, L. et al. 25th anniversary article: a decade of organic/polymeric photovoltaic research. Adv. Mater. 25, 6642-6671 (2013).
63 Masuko, K. et al. Achievement of more than 25\% conversion efficiency with crystalline silicon heterojunction solar cell. IEEE J. Photovoltaics 4, 1433-1435 (2014).

64 Kayes, B. M. et al. in Proc. 37th IEEE Photovoltaic Specialists Conference 4-8 (IEEE, 2011).

65 Keavney, C., Haven, V. \& Vernon, S. in Proc. 21st IEEE Photovoltaic Specialists Conference 141-144 (IEEE, 1990)

66 Geisz, J., Steiner, M., Garca, I., Kurtz, S. \& Friedman, D. Enhanced external radiative efficiency for $20.8 \%$ efficient single-junction gainp solar cells. Appl. Phys. Lett. 103, 041118 (2013).

67 Huber, W. H. \& Duggal, A. R. in Thin Films for Solar and Energy Technology Vol. 9177 (SPIE Proc, 2015).

68 Osborne, M. Hanergys solibro has $20.5 \%$ CIGS solar cell verified by NREL. Available at. http://www.pv-tech.org/news/hanergys_solibro_has_20.5_cigs_solar_cell_ver ified_by_NREL (2014).

69 Liu, M., Johnston, M. B. \& Snaith, H. J. Efficient planar heterojunction perovskite solar cells by vapour deposition. Nature 501, 395-398 (2013).

70 Chen, G. et al. Co-evaporated bulk heterojunction solar cells with $>6.0 \%$ efficiency. Adv. Mater. 24, 2768-2773 (2012)

71 Sun, Y. et al. Solution-processed small-molecule solar cells with $6.7 \%$ efficiency. Nat. Mater. 11, 44-48 (2012)

72 Xue, J., Rand, B. P., Uchida, S. \& Forrest, S. R. A hybrid planar-mixed molecular heterojunction photovoltaic cell. Adv. Mater. 17, 66-71 (2005).

73 Fleetham, T. B. et al. Efficient zinc phthalocyanine/c60 heterojunction photovoltaic devices employing tetracene anode interfacial layers. ACS Appl. Mater. Interfaces 6, 7254-7259 (2014)

74 Xiao, X., Bergemann, K. J., Zimmerman, J. D., Lee, K. \& Forrest, S. R. Small-molecule planar-mixed heterojunction photovoltaic cells with fullerene-based electron filtering buffers. Adv. Energy Mater. 4, 1301557 (2014).

75 Guo, X. et al. High efficiency polymer solar cells based on poly (3-hexylthiophene)/ indene-c 70 bisadduct with solvent additive. Energy Environ. Sci. 5, 7943-7949 (2012)

(i) This work is licensed under a Creative Commons Attribution 4.0 International License. The images or other third party material in this article are included in the article's Creative Commons license, unless indicated otherwise in the credit line; if the material is not included under the Creative Commons license, users will need to obtain permission from the license holder to reproduce the material. To view a copy of this license, visit http://creativecommons.org/licenses/ by/4.0/ 